\title{
Expression and Regulation of Retinoic Acid Receptor Responders in the Human Placenta
}

Reproductive Sciences

2018, Vol. 25(9) 1357-1370

(C) The Author(s) 2017

Article reuse guidelines:

sagepub.com/journals-permissions DOI: $10.1177 / 19337|9| 1774676$

journals.sagepub.com/home/rsx

@SAGE

\author{
Hanna Huebner, $\mathrm{PhD}^{\prime}$, Andrea Hartner, $\mathrm{PhD}^{2}$, Wolfgang Rascher, $\mathrm{MD}^{2}$, \\ Reiner Strick R., PhD', Sven Kehl, MD', Felix Heindl, MD', \\ David L. Wachter, MD ${ }^{3}$, Matthias W. Beckmann, MD', \\ Fabian B. Fahlbusch, MD ${ }^{2, *}$, and Matthias Ruebner, PhD $^{1, *}$
}

\begin{abstract}
Introduction: Retinoic acid (RA) signaling through its receptors (RARA, RARB, RARG, and the retinoic X receptor RXRA) is essential for healthy placental and fetal development. An important group of genes regulated by RA are the RA receptor responders (RARRESI, 2, and 3). We set out to analyze their expression and regulation in healthy and pathologically altered placentas of preeclampsia (PE) and intrauterine growth restriction (IUGR) as well as in trophoblast cell lines. Methods: We performed immunohistochemical staining on placental sections and analyzed gene expression by real-time polymerase chain reaction. Additionally, we performed cell culture experiments and stimulated Swan7I and Jeg-3 cells with different RA derivates and 2'-deoxy-5-azacytidine (AZA) to induce DNA demethylation. Results: RARRESI, 2, and 3 and RARA, RARB, RARG, and RXRA are expressed in the extravillous part of the placenta. RARRESI, RARA, RARG, and RXRA were additionally detected in villous cytotrophoblasts. RARRES gene expression was induced via activation of RARA, RARB, and RARG in trophoblast cells. RARRESI was overexpressed in villous trophoblasts and the syncytiotrophoblast from PE placentas, but not in IUGR without PE. Promoter methylation was detectable for RARRESI and RARB based on their sensitivity toward AZA treatment of trophoblast cell lines. Discussion: RARRESI, 2 and 3 are expressed in the functional compartments of the human placenta and can be regulated by RA. We hypothesize that the epigenetic suppression of trophoblast RARRESI and RARB expression and the upregulation of RARRESI in PE trophoblast cells suggest an involvement of environmental factors (eg, maternal vitamin $A$ intake) in the pathogenesis of this pregnancy complication.
\end{abstract}

\section{Keywords}

placenta, preeclampsia, RARRESI, RARRES2, RARRES3

\section{Introduction}

Vitamin A is commonly used as a therapeutic agent for the treatment of psoriasis or in cancer therapy as it is known to induce tumor cell apoptosis and inhibition of cell proliferation. ${ }^{1,2}$ Retinoic acid (RA), the active derivate of vitamin A, is essential for healthy development and growth during embryogenesis and especially controls cellular key processes like development, differentiation, proliferation, and apoptosis. ${ }^{3,4}$ Vitamin A deficiency negatively affects different metabolic pathways, leading to a disruption of organogenesis (e.g. brain, kidney). ${ }^{5-7}$ RAs function through binding to RA receptors (RARs) and retinoic $\mathrm{X}$ receptors (RXRs) which are part of the steroid/thyroid/vitamin D3 nuclear receptor family. ${ }^{8}$ Both RARs and RXRs can form hetero- or homodimers which bind to specific DNA elements and act as ligand-inducible transcription factors. ${ }^{9}$

Furthermore, RAs are important regulators of human trophoblast functions during placentation and throughout gestation. ${ }^{4}$ RAs control trophoblast differentiation as well as hormone production within the syncytiotrophoblast (SCT). ${ }^{10}$ Metabolic signaling pathways are regulated by RA-induced production and release of, for example, human chorionic gonadotropin or human placental lactogen. ${ }^{10-12}$ RAs and their transcription factors additionally influence vital processes involved in trophoblast invasion, fusion, proliferation, and differentiation. ${ }^{12-15}$

\footnotetext{
I Department of Gynecology and Obstetrics, University Hospital Erlangen, Friedrich-Alexander University Erlangen-Nuremberg, Erlangen, Germany

${ }^{2}$ Department of Pediatrics and Adolescent Medicine, Friedrich-AlexanderUniversity of Erlangen-Nuremberg, Erlangen, Germany

${ }^{3}$ Institute of Pathology, University Hospital Erlangen, Friedrich-AlexanderUniversity Erlangen-Nuremberg, Erlangen, Germany

* The authors are contributed equally.
}

Corresponding Author:

Hanna Huebner, Laboratory for Molecular Medicine, Department of Gynecology and Obstetrics, University Hospital Erlangen, Universitaetsstr, 21-23, 91054 Erlangen, Germany.

Email: hanna.huebner@uk-erlangen.de 
Retinoic acid receptor responders (RARRES1, 2 and 3) are a group of RA-inducible genes which were identified after stimulation of skin cell explants with the RARB-/G-specific retinoid Tazarotene (TAZA). ${ }^{2}$ RARRES1 (also known as Tazarotene-induced gene 1 (TIG1), Latexin-like (LXNL), or Phorbol Ester-induced gene 1 (PERG-1)) is a transmembrane protein with a glycosylation-signal and a hyaluronic acid binding motive. ${ }^{2}$ RARRES1 is proposed as a tumor suppressor gene, as it is often found downregulated in various carcinomas. ${ }^{16,17} \mathrm{It}$ was shown that RARRES1 is capable of inducing apoptosis and inhibiting proliferation and invasion of cancer cells. ${ }^{18-20}$ RARRES2 (also known as Tazarotene-induced gene 2 (TIG2), HP10433 or Chemerin) on the other hand, is predominantly found in its secreted form. ${ }^{21}$ It is a chemoattractant, which is expressed in immune cells as well as adipose tissue and induces the chemotactic recruitment of immune cells to inflammatory areas. ${ }^{21}$ High RARRES2 serum levels were shown to be associated with, for example, psoriasis, diabetes, or cardiovascular disease. ${ }^{22-24}$ While RARRES2 serum levels significantly increase during pregnancy, ${ }^{25}$ little is known about its role during human placentation. In cancer, RARRES2 facilitates cell migration and invasion and is correlated with inflammation. ${ }^{26-}$ ${ }^{28}$ In contrast, RARRES3 was shown to be a tumor-suppressor protein, which influences tumor cell metastasis by regulating cell adhesion and differentiation. ${ }^{29}$ Its expression is downregulated in breast and colorectal carcinomas and loss of RARRES3 correlates with the presence of lung or nodal metastasis. ${ }^{29,30}$

RARRES1, 2, and 3 were previously shown to be directly regulated by the binding of RAR/RXR heterodimers to RARE consensus sequences of the respective promotor region. ${ }^{2,24,31}$ Furthermore, genes linked with retinoid signaling, like RARB, cellular retinol-binding protein 1, and RARRES1, were associated with DNA methylation in various carcinomas, and correlation of RARB and RARRES1 methylation was demonstrated..$^{32,33}$ While there are no CpGs (Cytosine nucleotides followed by guanine) within the RARE consensus sequence, DNA methylation of the surrounding $\mathrm{CpG}$ islands seems to influence transcriptional regulation of RARRES and RAR gene expression. ${ }^{33-35}$ Interestingly, hypermethylation of RARB promotor regions were shown in term placental samples, indicating a diminished RA signaling in late gestation. ${ }^{36}$ However, little is known about the transcriptional regulation of RARRES1, 2, and 3 in human trophoblast cell lines and the effect of DNA methylation on this signaling pathway.

Tumorigenesis and placentation share similar processes for the induction of invasion, proliferation, and inhibition of apoptosis, including the transcriptional regulation of those mechanisms. ${ }^{37}$ It is believed that failures in these pathways could lead to placental dysfunctions like intrauterine growth restriction (IUGR) or preeclampsia (PE). ${ }^{38}$ Alternatively, pathologic induction of invasion and proliferation is conceived as a risk factor for the development of malignant transformations (e.g. choriocarcinoma ${ }^{39}$ ).

In order to investigate the impact of RA signaling during placentation, we set out to analyze the expression and regulation of RARRES1, 2, and 3 and their transcription factors RARA, RARB, RARG, and RXRA in healthy and pathologically altered placental tissue (PE/IUGR) and various trophoblast-like cell lines.

\section{Materials and Methods}

\section{Patient and Tissue Collection}

Human term placentas were obtained from healthy patients (n $=15)$ or pregnancies complicated by PE $(\mathrm{n}=3)$ and/or idiopathic IUGR $(n=3)$ after elective cesarean delivery, with " $n$ " representing different patients. The clinical data of all patients are summarized in Supplementary Table 1. The inclusion criteria for PE and IUGR were described earlier by Fahlbusch and Ruebner et al. ${ }^{40,41}$ Placental tissues were formalin-fixed and paraffin-embedded (FFPE) for histological staining or snap frozen in liquid nitrogen for RNA extraction within 1 hour after delivery.

\section{Ethical Approval}

Each participant signed a written informed consent. Handling of patients and tissues were approved by the Ethics Committee at the University of Erlangen-Nuremberg (No. 353_15B).

\section{Immunohistochemical Staining}

Human FFPE placental sections were deparaffinized using Roti-Histol (Carl Roth, Karlsruhe, Germany) and rehydrated in a series of descending ethanol concentrations. Immunohistochemical staining was performed using the labeled streptavidin-biotin (LSAB) + horseradish peroxidase (HRP) kit (Agilent, Hamburg, Germany) according to the manufacturer's instructions. Mouse anti-human RARRES1 (1:6000 ab92884; Abcam, Cambridge, UK), rabbit anti-human RARRES2 (1:100 ab729065; Abcam), rabbit anti-human RARRES3 (1:50 ab77010; Abcam), rabbit anti-human RAR $\alpha$ (1:250 sc551; Santa Cruz, Heidelberg, Germany), rabbit anti-human RAR $\beta$ (sc552 1:50; Santa Cruz), rabbit anti-human RAR $\gamma$ (sc550 1:50; Santa Cruz), and rabbit anti-human RXR $\alpha$ (1:50 ABIN685702; antibo dies-online.com, Aachen, Germany) antibodies were used. Nuclei were stained with hematoxylin. Images were obtained using the Nikon Eclipse 80i microscope (Nikon, Duesseldorf, Germany).

\section{Isolation of Primary Trophoblast Cells}

Primary trophoblast cells were isolated from 10 individual healthy term placentas as well as placentas complicated by PE $(n=3)$, PE/IUGR $(n=3)$, or IUGR $(n=7)$. The trypsin-DNase-Dispase/Percoll method was used, as previously described. ${ }^{42}$ Before RNA isolation, the primary trophoblast cells were cryopreserved in liquid nitrogen. The percentage of trophoblastic cells (86.6\%-90\%) was determined by fluorescence activated cell sorting (FACSCalibur; BD Biosciences, Heidelberg, Germany).

\section{Cell Culture}

Stimulation experiments were performed using the choriocarcinoma cell line Jeg-3 and the first trimester cell line Swan71. 
The Swan71 cells were a kind gift from Dr G. Mor, Department of Obstetrics, Gynecology and Reproductive Sciences, Reproductive Immunology Unit, Yale University School of Medicine, New Haven, USA. ${ }^{43}$ The cell lines were cultivated in DMEM: F12 phenol-red free (high glucose; Thermo Fischer, Darmstadt, Germany) supplemented with $10 \%$ fetal calf serum (FCS; Thermo Fischer), $100 \mathrm{U} / \mathrm{mL}$ penicillin, and $100 \mu \mathrm{g} / \mathrm{mL}$ streptomycin (Sigma-Aldrich, Taufkirchen, Germany). Swan71 cells were additionally supplemented with $0.1 \mathrm{mM}$ nonessential amino acids (NEAAs; Sigma-Aldrich). Furthermore, RNA was extracted from BeWo and JAR cells. BeWo cells were grown in DMEM: F12 phenol-red free (high glucose; Thermo Fischer) supplemented with $10 \%$ FCS (Thermo Fischer), $100 \mathrm{U} / \mathrm{mL}$ penicillin, and $100 \mu \mathrm{g} / \mathrm{mL}$ streptomycin ( $1 \%$ P/S; Sigma-Aldrich). JAR cells were cultivated in RPMI 1640 media (Thermo Fischer) with $10 \%$ FCS, 10 mM Hepes (Sigma-Aldrich), $2 \mathrm{mM}$ L-glutamine (Sigma-Aldrich), and 0.1 mM NEAAs (Sigma-Aldrich).

For treatment with 2'-deoxy-5-azacytidine (AZA; SigmaAldrich) or RA, cells were seeded at a density of $3 \times 10^{4}$ / $\mathrm{mL}$ in a 12-well cell culture plate. Cells were cultivated in $2.5 \%$ (Swan71) or 5\% (Jeg-3) charcoal-treated fetal bovine serum (Thermo Fisher) 24 hours prior to and during stimulation. Cells were treated with $1.0 \mu \mathrm{M}$ of all-trans-RA (ATRA; Biomol, Hamburg, Germany) of the RAR $\beta-/ \gamma$-specific RA derivate TAZA (Sigma-Aldrich) and $200 \mathrm{nM}$ of the RAR $\alpha$ agonist Am580 (Tocris; Lille Cedex, France) for 48 hours. AZA was used in a concentration of $1.0 \mu \mathrm{M}$ for 72 hours. For a specific inhibition of RA stimulation, different RAR antagonists were used. Therefore, cells were treated with CD2665 (RAR $\beta / \gamma$ antagonist) or ER50891 (RAR $\alpha$ antagonist) at concentrations between 0.5 and $8.0 \mu \mathrm{M}$ for 48 hours. Every 24 hours, the medium was changed and the stimulation was repeated. The specificity of Am580, TAZA, ATRA, and the antagonists were commercially and/or experimentally tested beforehand by others. ${ }^{5,44-49}$ Agonists and antagonists were used as described elsewhere, ${ }^{2,50,51}$ and different concentrations as well as time points were tested by us beforehand (data not shown). We chose to conduct our experiments at 48 hours, as it represents the time point with the highest RA-induced increase of gene expression. All experiments were performed with 6 or 9 biological replicates from different passages of each cell line. The different RAs and antagonists were either solubilized in ethanol or DMSO. Thus, cells were treated with ethanol and/or DMSO in the respective amounts preanalytically. We found no differences in the gene expression of target genes and housekeepers with and without ethanol and/or DMSO. Hence, we used the "no treatment" condition (i.e. without ethanol/DMSO) as a control for our final experiments.

\section{Extraction of RNA}

As previously described, ${ }^{41}$ RNA was isolated from placental tissues, primary trophoblast cells, and cultivated cell lines (Swan71, Jeg-3, JAR and BeWo) using peqGold Trifast (Peqlab, Erlangen, Germany). Before complementary DNA
(cDNA) synthesis, RNA was treated with DNase I (Roche, Mannheim, Germany). The cDNA was generated using the high-capacity cDNA Reverse Transcription kit (Thermo Fisher) in a thermal cycler (ABI2720) for 2 hours at $37^{\circ} \mathrm{C}$.

\section{Quantitative Real-Time Polymerase Chain Reaction}

Quantification of RARRES1, 2, and 3 (semiquantitative) as well as RARA, B, and G (full quantitative) cDNA was performed by quantitative real-time polymerase chain reaction (qRT-PCR). Forward and reverse primer of $10 \mathrm{nM}$ and cDNA of $40 \mathrm{ng}$ were used and quantification analyzed by SYBR-green-based technology (SYBR select master mix; Thermo Fischer). The expression was normalized using 18SrRNA and Glyceraldehyde 3-phosphate dehydrogenase (GAPDH) as reference genes. Due to lack of differences, only 18 SrRNA data are presented. Primer sequences are listed in Supplementary Table 2. For RAR and RXR, a full quantitative qRT-PCR analysis using plasmids with the respective region of the gene of interest was performed in order to calculate the molecules per ng cDNA. This allowed the comparison of different genes within 1 cell line. The ng cDNA content was calculated using a standard curve and $R A R / R X R$ cDNA plasmids. Semiquantitative analysis was performed using the $\Delta \Delta \mathrm{C}_{\mathrm{T}}$ method and values were normalized to the untreated control. All semi- and full quantitative qRT-PCR analyses were performed in duplicates.

\section{Statistical Analysis}

All data are presented as mean \pm standard error of the mean (SEM). Differences between the subgroups were analyzed using the Mann-Whitney $U$ test (SPSS Version 21; IBM Inc., Armonk, USA). $P$ values of $\leq .05$ were considered statistically significant.

\section{Results}

\section{Expression of RARRESI, 2, and 3 in Different Compartments of the Human Placenta}

In order to determine whether the RA responsive genes RARRES1, 2, and 3 are expressed in human placental tissues and trophoblastic cell lines (Swan71, Jeg-3, JAR and BeWo), we performed qRT-PCR analysis of RNA isolated from tissues and cell lines (Figure 1A) and immunohistochemical staining of third-trimester placental FFPE sections (Figure 1B).

In comparison to placental biopsies, significantly higher RARRES1 and RARRES3 expression was detected in isolated villous cytotrophoblasts (trophobl; Figure 1A, a and c; $P<.05$ ). Furthermore, the first-trimester cell line Swan71 showed a significantly higher expression of RARRES1,2, and 3 compared to placental tissues (Figure 1A, a-c; $P<.005$ ). Expression of RARRESI was significantly reduced $(P<.005)$ in the choriocarcinoma cell lines Jeg-3, JAR, and BeWo. Similar results were obtained for RARRES2 expression in Jeg-3 and BeWo cells $(P<.05)$ and for RARRES3 in Jeg-3 and JAR cells $(P<$ $.05)$. In contrast, a significantly higher expression was detected for RARRES2 in the JAR cell line (3-fold, $\mathrm{p}<0.05$ ) and for 


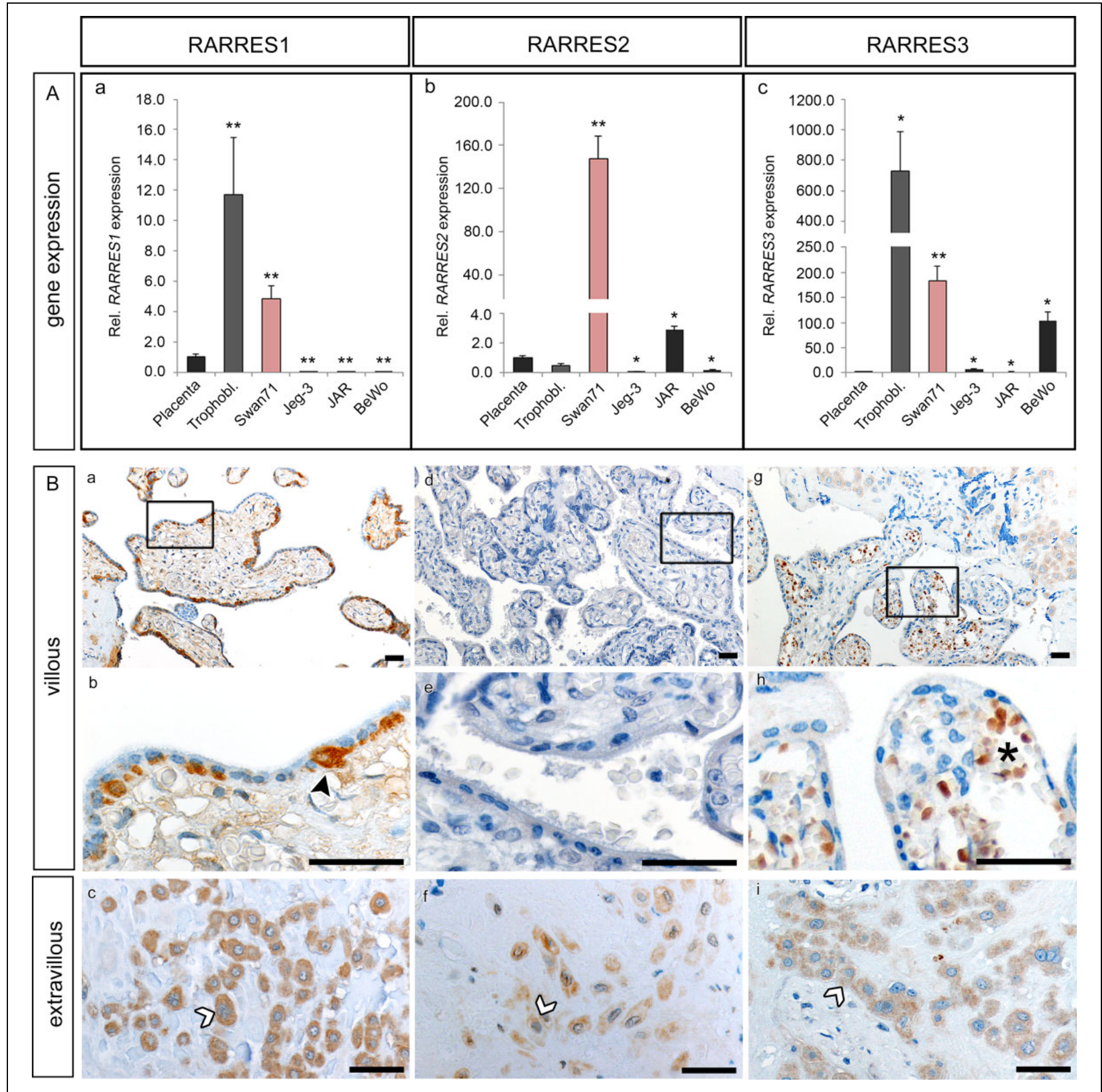

Figure I. Expression of RARRESI, 2, and 3 in the human placenta. A, RARRESI (a), RARRES2 (b), and RARRES3 (c) gene expression (mean and SEM) was analyzed by qRT-PCR in placental tissue (biopsies from $n=15$ different placentas), primary trophoblast cells (Trophobl., isolated from $\mathrm{n}=5$ different placentas), the first-trimester cell line Swan7I $(\mathrm{n}=9)$, and the choriocarcinoma cell lines Jeg-3 $(n=6), J A R(n=9)$, and BeWo $(n$ $=6$ ). The level of significance is indicated as follows: $* P \leq .05$ and $* * P \leq .005$, relative to placental tissue. $\mathrm{B}$, Protein expression of RARRESI (a-c), RARRES2 ( $\mathrm{d}-\mathrm{f})$, and RARRES3 ( $\mathrm{g}-\mathrm{i})$ was analyzed by immunohistochemical staining of placental FFPE sections. RARRESI-positive VTs are indicated by black arrowheads (b) and RARRESI-, 2-, or 3-positive EVTs by white arrowheads (c, $\mathrm{f}$, and i). The asterisk marks a fetal vessel (h). Black bars define $100 \mu \mathrm{m}$. FFPE indicates formaldehyde-fixed and paraffin-embedded; qRT-PCR, quantitative real-time polymerase chain reaction; RARRES, retinoic acid receptor responder; SEM, standard error of the mean.

RARRES3 in BeWo cells (100-fold; $P<.05)$, when compared to normal third-trimester placental tissue.

Within the analyzed placental FFPE sections, we detected RARRES1 protein expression in the villous (Figure 1B, a and b) as well as the extravillous (Figure 1B, c) part of the human placenta. Villous RARRES1 protein was mainly expressed in villous trophoblasts (VTs), which were neighboring the SCT border (Figure 1B, b, black arrowhead). The RARRES1 


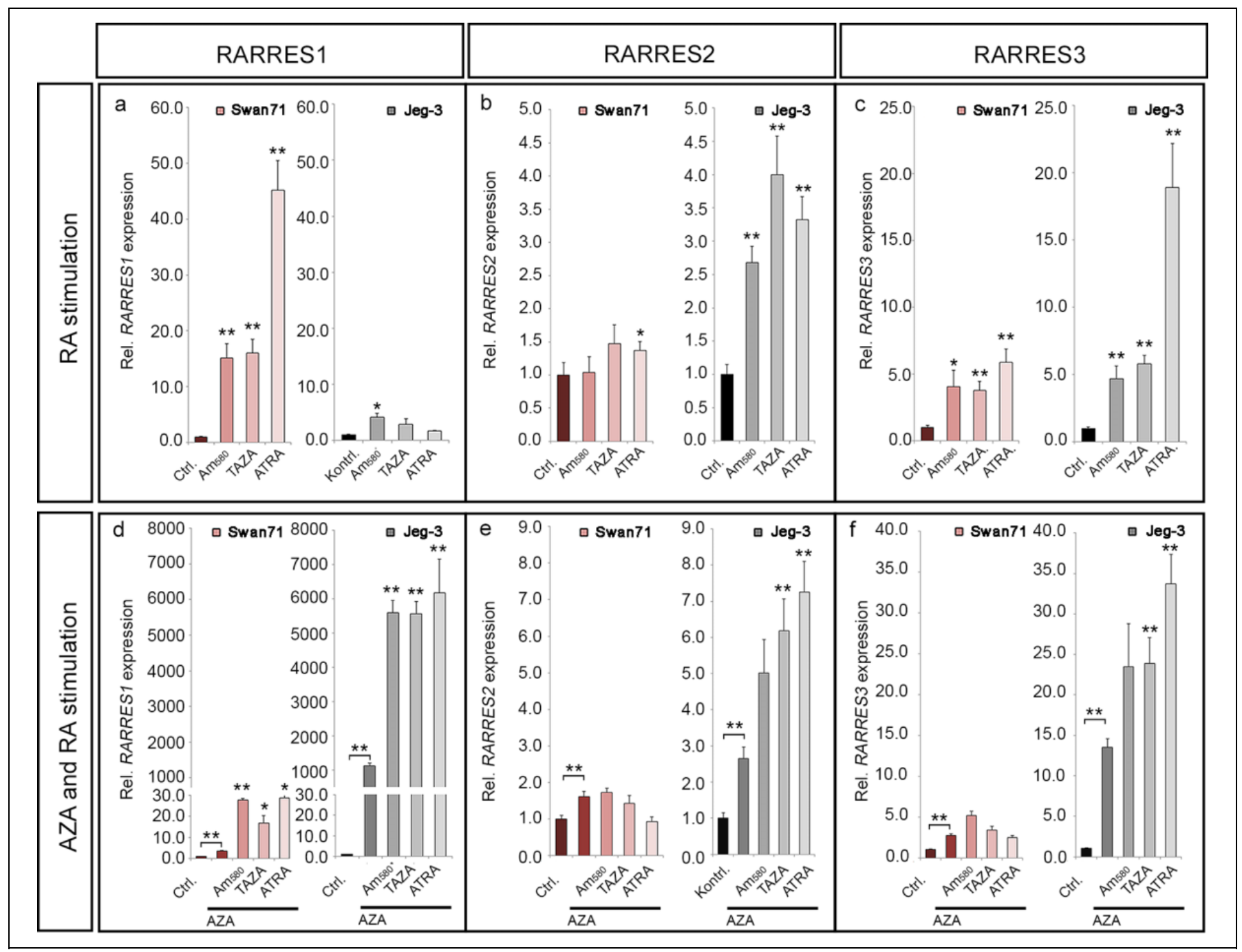

Figure 2. Expression of RARRESI, 2, and 3 after RA and AZA treatment. Swan7I and Jeg-3 cells were stimulated with different RAs (Am580, TAZA and ATRA) and gene expression (mean and SEM) of RARRESI (a), RARRES2 (b), and RARRES3 (c) was analyzed by qRT-PCR. Swan7I and Jeg-3 cells were additionally treated with AZA to induce DNA demethylation and stimulated with RAs to induce gene expression even further (d-f). The graph shows the relative fold change of gene expression normalized to the untreated control (Ctrl.). The level of significance is indicated as following: $* P \leq .05$ and $* * P \leq .005$, relative to Ctrl. or solely AZA treated cells, $n=6$. ATRA indicates all-trans-retinoic acid; AZA, 2'-deoxy-5-azacytidine; qRT-PCR, quantitative real-time polymerase chain reaction; RA, retinoic acid; RARRES, retinoic acid receptor responder; SEM, standard error of the mean; TAZA, Tazarotene.

staining was cytosolic and membranous for both extravillous trophoblasts (EVTs) and VTs. On the other hand, only EVTs showed a RARRES2-positive staining (Figure 1B, f). In addition, EVTs did express RARRES3 (Figure 1B, g, and i), while the VTs and the SCT did not show any RARRES2 or 3 staining (Figure 1B, d, e, and g, h, respectively). Additionally, fetal blood cells were RARRES3 positive (Figure 1B, h, black asterisk).

\section{Expression of RARRESI, 2, and 3 can be Induced by RAs and AZA Treatment}

As the highest expression for all 3 RARRES genes was detected in the Swan71 cell line and the lowest expression in the choriocarcinoma cell line Jeg-3, these cell lines were chosen for further stimulation experiments. Our primary objective was to analyze whether RARRES1, 2, and 3 expression could be increased by treatment with different RAs. On the one hand, cultured cells were treated with the RARA-specific agonist Am580, with the RA derivate TAZA (RARB- and RARG-specific) or with ATRA (RARA-, RARB-, and RARG-specific; Figure 2a-c) and on the other hand additional treatment with AZA was performed to obtain global DNA demethylation (Figure 2d-f). In the Swan71 cell line significant stimulation of RARRES1 and RARRES3 expression was obtained after treatment with Am580, TAZA, and ATRA (Figure 2a and c). Expression of RARRES2 and 3 increased significantly after stimulation of Jeg-3 cells with all RA derivates (Figure 2b; $P$ $<.005)$. No increase was detected in RARRES1 gene expression. In order to investigate whether the lack of stimulation capacity was caused by DNA methylation, we treated the cells 


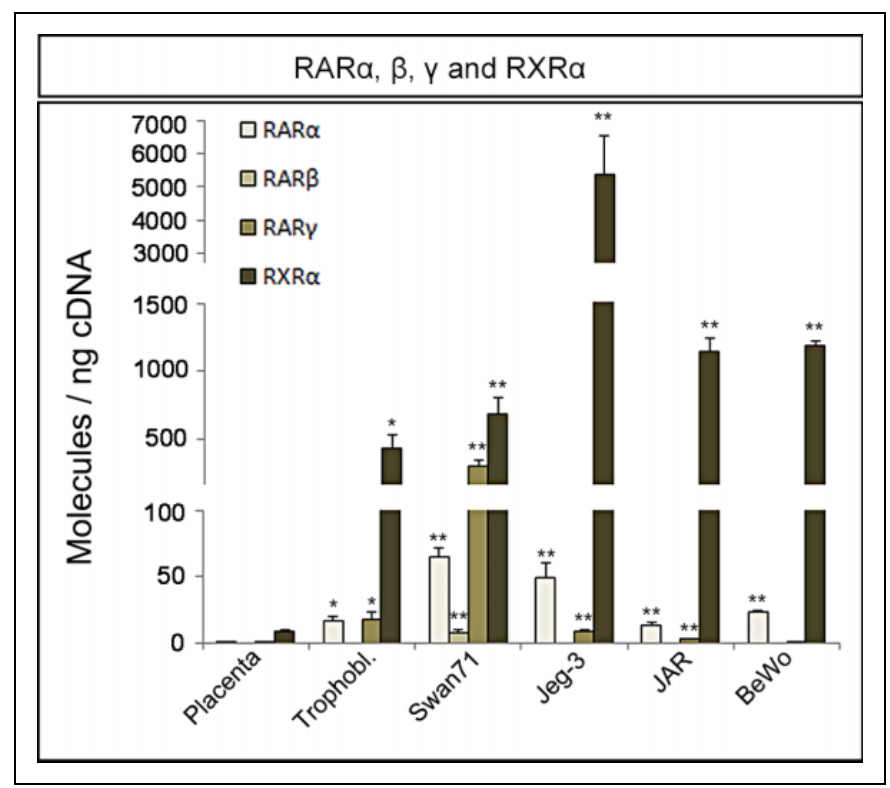

Figure 3. Expression of $\operatorname{RAR} \alpha, \beta, \gamma$, and $R X R \alpha$ in the human placenta. Molecules per ng cDNA of RAR $\alpha, \beta, \gamma$, and $\operatorname{RXR} \alpha$ were quantified by qRT-PCR analysis. The RNA of placental tissue $(n=4)$, primary trophoblast cells $(n=6)$, Swan7I $(n=10)$, Jeg-3 $(n=9)$, JAR $(n=9)$, and BeWo $(n=9)$ cells was isolated and analyzed. The level of significance is indicated as follows: $* P \leq .05$ and $* * P \leq .005$, relative to placental tissue. cDNA indicates complementary DNA; qRT-PCR, quantitative real-time polymerase chain reaction; RAR, retinoic acid receptor.

with AZA before RA stimulation. Gene expression of all three genes was induced significantly after AZA treatment $(P<.005$; Figure 2d-f). Furthermore, AZA treatment of Jeg-3 cells facilitated the stimulation of RARRES1 expression by RA (Figure $2 \mathrm{~d} ; P$ $<.005$ ), which could not be achieved without AZA (Figure 2a).

\section{Retinoic Acid Receptor A, G, and RXRA are Highly Expressed in Placental Tissue}

Gene expression of RARRES1,2, and 3 was induced by all 3 RA derivates (Am580, TAZA, and ATRA) in Swan71 and Jeg3 cells. In order to investigate whether RAs could stimulate RARRES expression via RAR transcription factors, we analyzed the expression of $R A R A, R A R B$, and $R A R G$ as well as $R X R A$ in placental tissue and trophoblastic cell lines (Figure 3) and performed immunohistochemical staining on placental FFPE sections (Figure 4). Analysis of gene expression by qRT-PCR showed a significantly higher expression of $R A R A$, $R A R G$, and $R X R A$ in primary trophoblast cells compared to total placental tissue (Figure 3; $P<.05$ ). Overall, we detected the highest number of molecules per ng cDNA for RXRA (Figure 3). The lowest expression was found for $R A R B$, which was even below the lower limit of detection in placental tissue and primary trophoblast cells (Figure 3).

Using immunohistochemical staining, we detected RARA, $R A R G$, and RXRA in the villous as well as the extravillous compartments of the human placenta (Figure 4a-c and g-l). All
3 RARs were expressed in the nuclei of EVTs and VTs (Figure 4b, h, and k; black asterisks; and c, i, and l, black arrowheads). Additionally, RARA and RARG were expressed in several nuclei of the SCT (Figure $4 \mathrm{~b}$ and $\mathrm{h}$, black arrowheads), while others were negative for the receptors (Figure 4, white arrowheads). In contrast, RARB could not be detected in the villous part of the human placenta but showed a cytoplasmic staining in the EVTs (Figure 4d-f).

\section{Expression of RARA, RARB, and RARG Significantly Increases After AZA Treatment}

Overall, RARA, RARG, and RXRA could be detected in the same placental compartments and cell lines as RARRES1, 2, and 3 and thus might function as transcription factors to increase RARRES gene expression. However, earlier publications showed that $R A R B$ is expressed and is an active transcription factor under RA stimulation only. ${ }^{34,52,53}$ Moreover, it is well-known that transcriptional expression of $R A R B$ is controlled by epigenetic regulation. ${ }^{54-56}$ Consequently, we set out to analyze $R A R A, R A R B, R A R G$, and $R X R A$ expression under RA and AZA treatment. Interestingly, when stimulated with Am580, the expression of all analyzed receptors increased significantly in Swan71 cells (Figure 5a-d). Nevertheless, the strongest effect could be detected for $R A R B$ (up to 20-fold increase; Figure $5 \mathrm{~b} ; P<.005$ ), regardless of the RA derivative used. A slight, but significant increase in $R A R A$ as well as $R A R B$ expression could additionally be shown in Jeg-3 cells (Figure $5 \mathrm{a}$ and $\mathrm{b}$ ). This increase in $R A R B$ expression was more pronounced after AZA treatment (up to 35-fold; Figure 5f). AZA itself induced the expression of $R A R A, R A R B$, and $R A R G$, while the expression of $R X R A$ decreased significantly in AZAtreated Jeg-3 cells (Figure 5e-h; $P<.05$ ).

\section{Expression of RARRESI, 2, and 3 can be Blocked by RAR Antagonists}

To investigate the specific regulation of RARRES1, 2, and 3 expression through RARA, B, or G, we performed additional cell culture experiments and used specific receptor antagonists to inhibit RA stimulation. CD2665 is a specific blocker of RARB and RARG, while ER50891 is a specific RARA antagonist. We treated Swan71 and Jeg-3 cells with CD2665 and ER50891 in different concentrations and added the RAs (Am580, TAZA, and ATRA) to increase RARRES1, 2, and 3 expression (Figure 6 and Supplemental Figure 1). In Swan71 cells RA-induced gene expression of RARRES1 and 3 decreased after treatment with CD2665 and ER50891 (Figure $6 \mathrm{~A}$ and $\mathrm{C}$, a-d). As shown before, RARRES2 expression could not be increased with Am580, TAZA, or ATRA and, consequently, there was almost no decline detected after inhibition with CD2665 or ER50891 (Figure 6B, a-d). Only the combination of both inhibitors in their highest concentration showed a significant inhibition of RARRES2 under TAZA treatment (Figure 6 and Supplemental Figure 1B, c). After treatment of Jeg-3 cells with AZA for demethylation, higher concentrations 


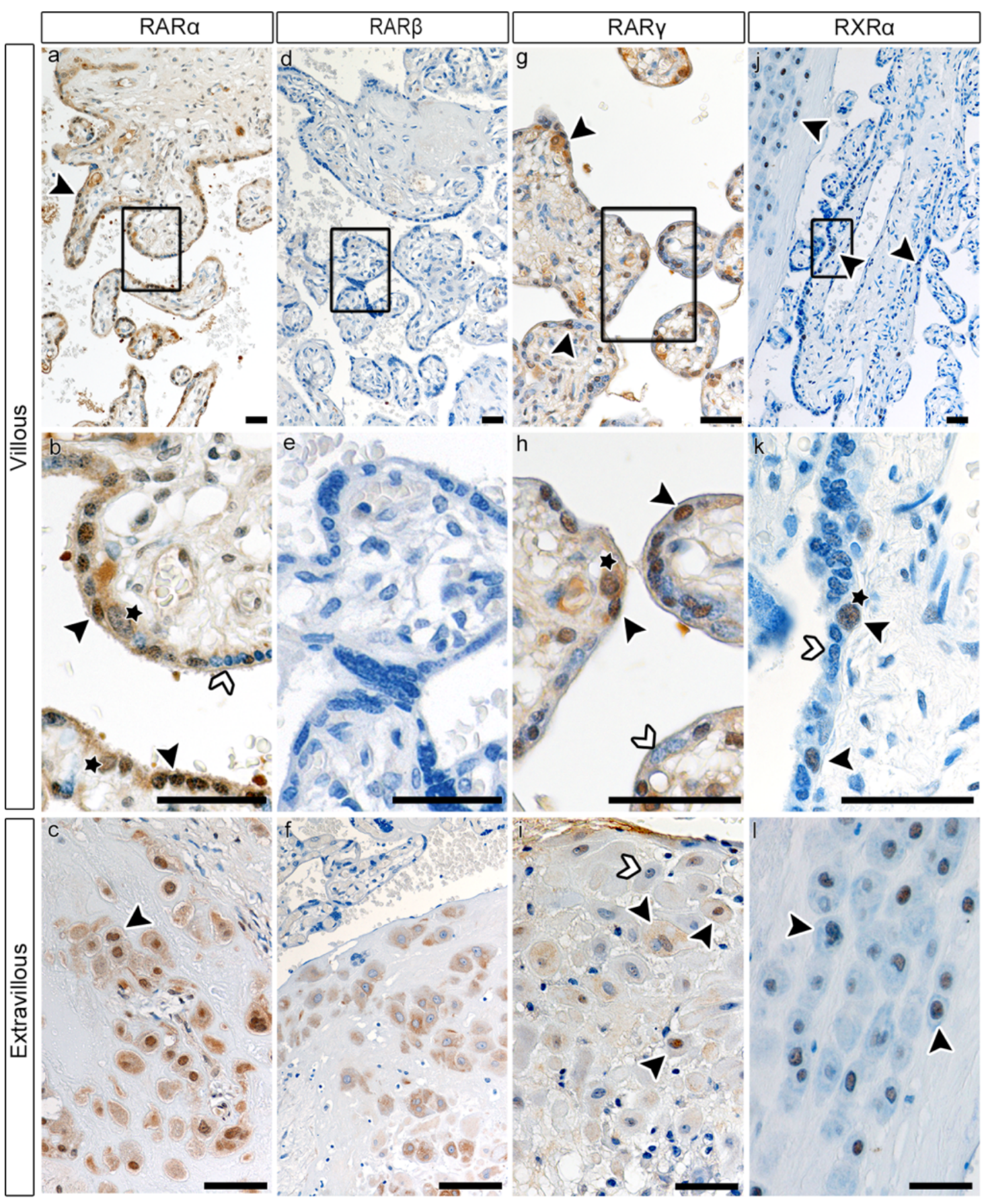

Figure 4. Expression of $\operatorname{RAR} \alpha, \beta, \gamma$, and $\mathrm{RXR} \alpha$ protein in human placental tissue. Protein expression of RAR $\alpha$ (a-c), RAR $\beta$ (d-f), RAR $\gamma(\mathrm{g}-\mathrm{i})$, and $R X R \alpha(j-l)$ was analyzed by immunohistochemical staining of placental FFPE sections. Black arrowheads indicate positive nuclei; white arrowheads show negative ones (a-l). Asterisks mark VTs (b, h, and k). Black bars define $100 \mu \mathrm{m}$. FFPE indicates formaldehyde-fixed and paraffinembedded; RAR, retinoic acid receptor; VT, villous trophoblast.

of the RARB/G-specific antagonist were necessary to induce a significant reduction in RARRES1, 2, or 3 expression (Figure 6A-C and Supplemental Figure 1, g; $P<.005$ ). This was in concordance with the detected increase in $R A R B$ and $R A R G$ expression after AZA treatment (Figure $5 \mathrm{f}$ and $\mathrm{g}$ ). Overall, the stimulation of gene expression could be significantly inhibited using RARA- as well as RARB/G-specific antagonists.

\section{Expression of RARRESI Increased in Villous Trophoblasts of PE Placentas}

As RARRES1, 2, and 3 were found to be expressed in the human placenta and seemed to be influenced by RA stimulation, we were interested in their expression pattern in different placental pathologies. Therefore, we analyzed gene and protein 


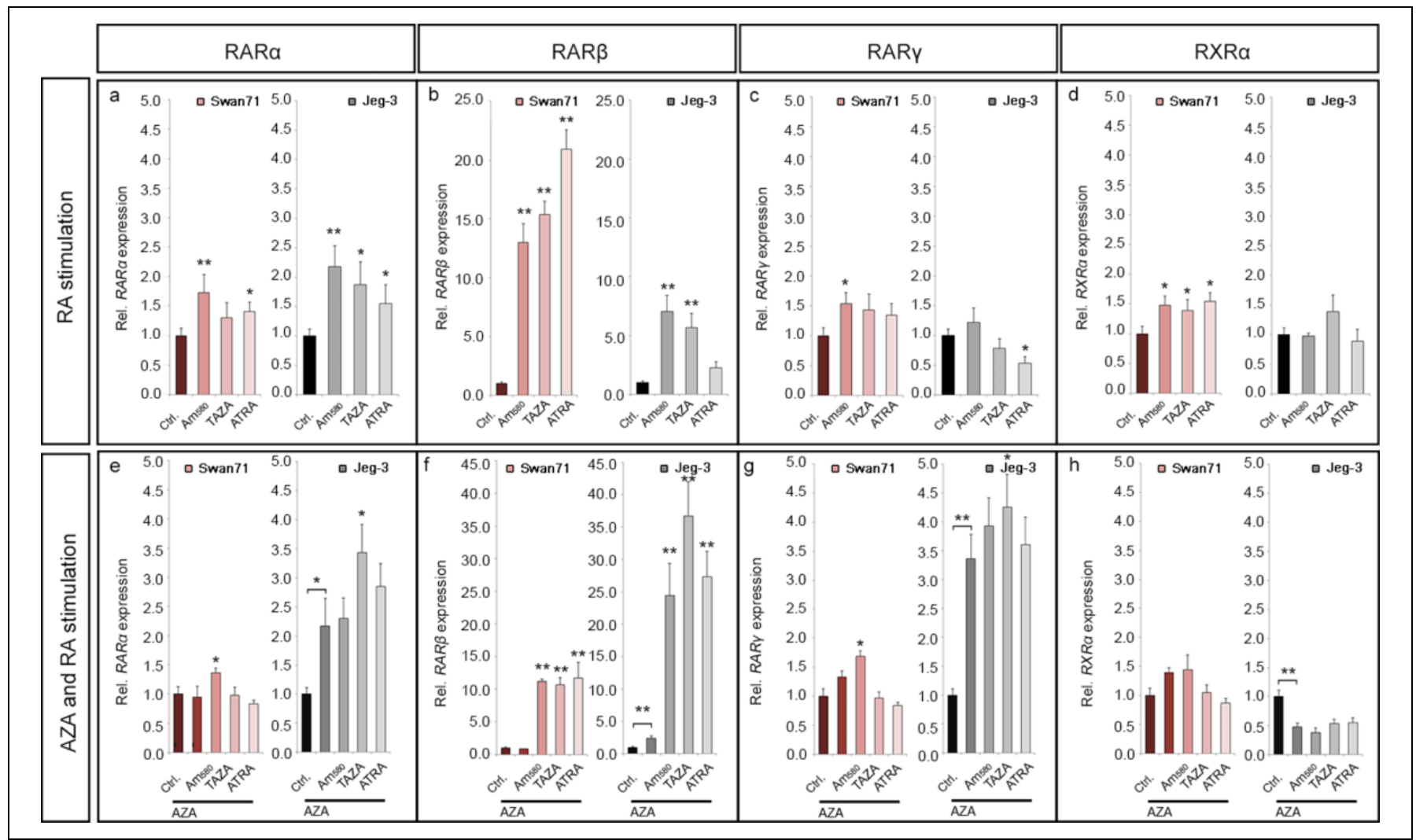

Figure 5. Expression of $R A R \alpha, \beta, \gamma$, and $R X R \alpha$ under RA and AZA treatment. Swan7I and Jeg-3 cells were treated with different RAs alone (Am580, TAZA, and ATRA; a-d) or together with AZA (e-h). Graphs show the relative fold change of gene expression (mean and SEM) to the untreated control (Ctrl.). The level of significance is indicated as follows: $* P \leq .05$ and $* * P \leq .005$, relative to Ctrl. or solely AZA treated cells, $n$ =6. ATRA indicates all-trans-retinoic acid; AZA, 2'-deoxy-5-azacytidine; RA, retinoic acid; RAR, retinoic acid receptor; SEM, standard error of the mean; TAZA, Tazarotene.

expression in placentas from healthy pregnancies and from the pregnancy complications PE and IUGR. Even though we detected a significant decrease in RARRES2 gene expression in placentas complicated by PE or IUGR, we did not observe a decrease in protein expression by immunohistochemical staining of FFPE sections (data not shown). We also did not detect any differences regarding RARRES3 gene and protein expression (data not shown). On the other hand, we found an increase in RARRES1 protein expression by immunohistochemical staining of sections from placentas complicated by PE/IUGR when compared to control placentas (Figure 7a-d). In contrast to healthy controls, PE/IUGR placenta sections showed a specifically strong RARRES1 staining within the SCT (Figure 7c). Based on the observation that this increase was only within the villous part of the placenta, we isolated primary villous trophoblast cells from control placentas and placentas complicated by PE and/or IUGR and analyzed RARRESI gene expression. RARRES1 was significantly increased in PE/IUGR (5-fold) and PE (3-fold) trophoblast cells (Figure $7 \mathrm{~g} ; P<.05$ ), while there was no difference in IUGR alone. Similarly to primary trophoblasts from PE/IUGR placentas, we found a 5-fold higher RARRES1 expression in the first-trimester cell line Swan71 compared to healthy placental tissue (Figure 1). Thus, we were further interested in the protein expression pattern of
RARRES1 in first-trimester placental tissue. Immunohistochemical staining of first-trimester FFPE sections revealed a strong RARRES1 expression in the villous (Figure 7e) and extravillous (Figure $7 \mathrm{f}$ ) compartment. Similarly to PE/IUGR placentas, RARRES1 staining was specifically strong in the SCT (Figure 7e).

\section{Discussion}

We observed high expression of RARRESI in third-trimester placental tissue, primary villous cytotrophoblasts as well as in the first-trimester cell line Swan71 and determined a significant induction of RARRES1 by different RAs. Thus, we assume that RARRES1 might exert important functions during placental development and for the maintenance of gestation. RARRES1 is known to be significantly downregulated in cancer cell lines or tissues like breast, prostate, or colorectal carcinomas. ${ }^{17,57,58}$ Here, we showed a significant decrease in RARRES1 expression in choriocarcinoma cell lines, which was restored by demethylation via AZA treatment. The RARRES1 promotor is known to be hypermethylated in various cancers. ${ }^{59,60}$ Therefore, we assume that an increased promotor methylation in choriocarcinomas might have contributed to the low RARRES1 expression detected. This might further go in line 


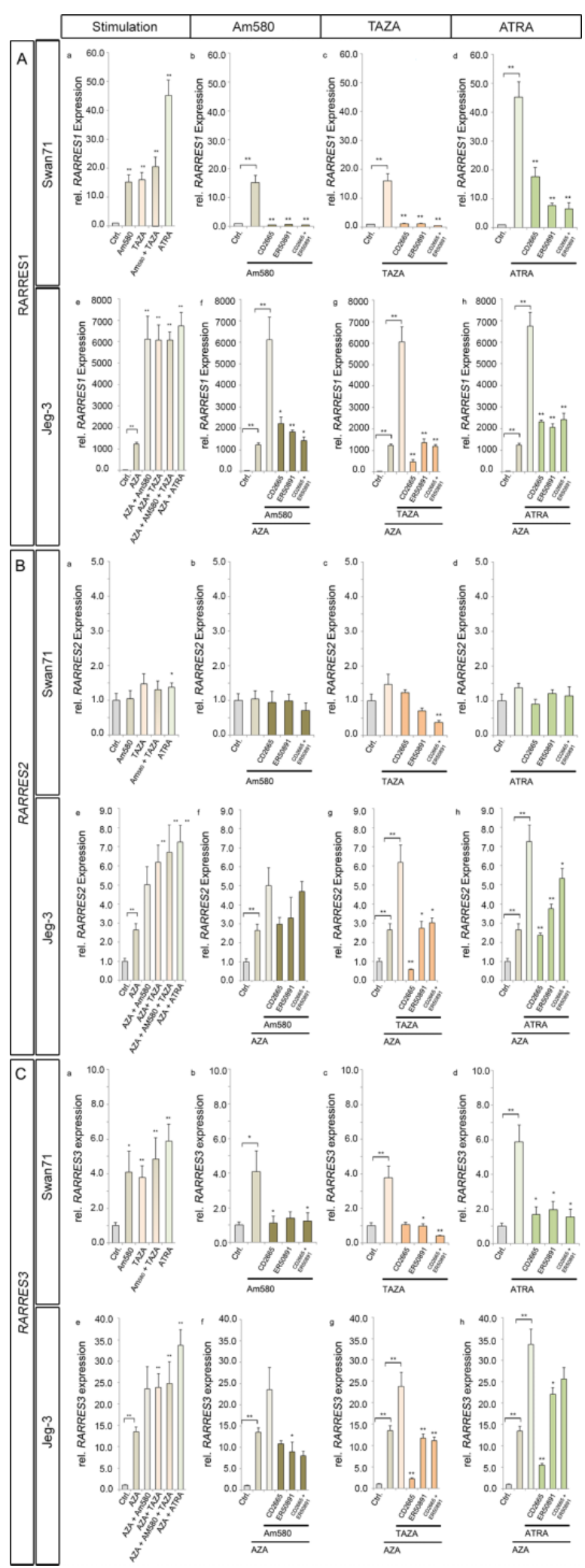

Figure 6. The RAR-dependent expression of RARRESI, 2, and 3. Swan7I cells were cultivated and treated with RAR antagonists (CD2665: RARB/G antagonist; ER5089I: RARA antagonist) and agonists (Am580, TAZA, and ATRA; A, B, and C, a-d). CD2665 and ER5089I were used in the most effective concentration of $2.0 \mu \mathrm{M}$. Additionally, Jeg-3 cells were pretreated with AZA and cocultured with CD2665 (8.0 $\mu \mathrm{M})$ or ER5089I (2.0 $\mu \mathrm{M})$ as well as Am580, TAZA, and ATRA (A, B, and C, e-h). The relative gene expression of RARRESI (A), RARRES2 (B), and RARRES3 (C) was quantified by qRT-PCR (mean and SEM). If not marked different, the level of significance is indicated as follows: $* P \leq .05$ and $* * P \leq .005$, relative to untreated $C$ trl. or solely $A Z A$ treated cells, $n=6$. ATRA indicates all-trans-retinoic acid; AZA, 2'-deoxy-5-azacytidine; qRT-PCR, quantitative real-time polymerase chain reaction; $R A$, retinoic acid; RAR, retinoic acid receptor; RARRES, retinoic acid receptor responder; SEM, standard error of the mean; TAZA, Tazarotene. 


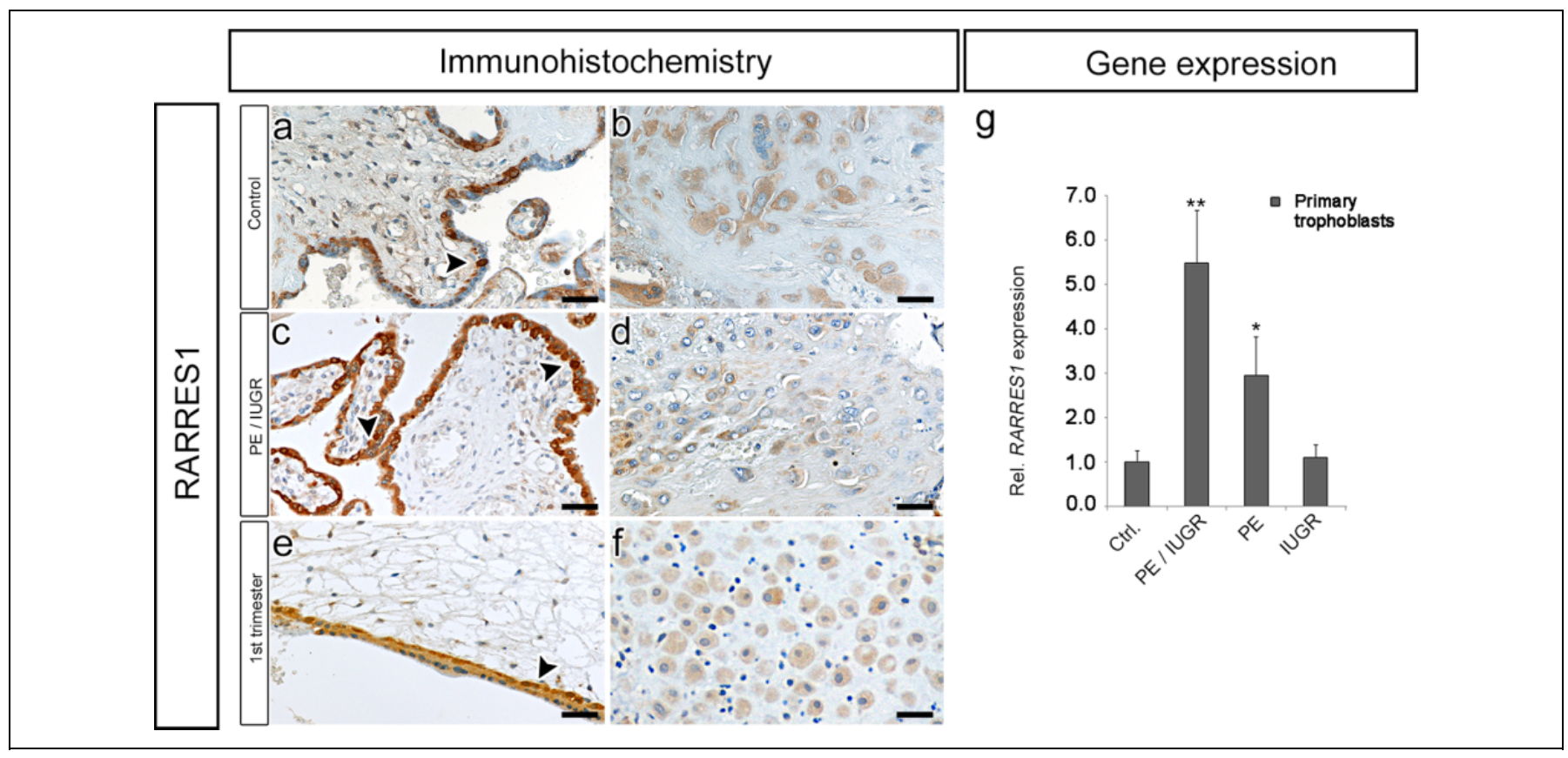

Figure 7. Expression of RARRESI in control and PE/IUGR placentas. Immunohistochemical staining of RARRESI was performed on FFPE sections from healthy control ( $a$ and b), PE/IUGR ( $c$ and d), and first-trimester (e and f) placentas. Black arrowheads mark VTs. Black bars define I00 $\mu \mathrm{m}$. Primary villous trophoblast cells were isolated from healthy (Ctrl., $n=10)$, PE/IUGR $(n=3), P E(n=3)$, and IUGR $(n=37)$ placentas. RNA was extracted and quantified by qRT-PCR analysis (g; mean and SEM). Visualized are the fold changes relative to control trophoblast cells and the significance is marked with stars $(* P \leq .05$ and $* * P \leq .005)$. FFPE indicates formaldehyde-fixed and paraffin-embedded; IUGR, intrauterine growth restriction; PE, preeclampsia; qRT-PCR, quantitative real-time polymerase chain reaction; RARRES, retinoic acid receptor responder; SEM, standard error of the mean; VT, villous trophoblast.

with the observed failure of inducing RARRES1 expression by RA in Jeg-3 cells. After AZA treatment, RARRES1 expression was significantly increased in Jeg-3, which further emphasizes our hypothesis of DNA methylation and subsequently reduced capability to induce RARRES1 expression in these cells. In breast cancer as well as prostate cancer cell lines, it was shown that RARRESI can significantly decrease invasion and proliferation. ${ }^{18,57}$ Thus, we hypothesize that RARRES1 might slow down invasion and migration of EVTs in terminal placentas and reduce the proliferation of VTs. A decrease in proliferation is necessary for the differentiation of VTs and fusion into the SCT. Supportive of this idea, we detected a high RARRES1 protein expression especially in VTs with spatial fusion capacity, that is, in close proximity to the SCT. As RARRES1 expression was significantly increased in isolated VTs complicated by $\mathrm{PE}$, we assume that high RARRES1 expression might negatively influence fusion capacity or increase the presence of apoptotic bodies. This hypothesis might go in line with the observed increase of a VT cell count, decreased number of fusions, and decreased expression of the fusogenic Syncytin 1 in PE placentas. ${ }^{42,61,62}$ On the other hand, re-expression of RARRES1 in the breast cancer cell line SUM 149 could promote apoptosis, which might be indicative of a similar association in PE placentas. ${ }^{16}$ It is well-known that syncytial knots representing apoptotic cells are increased in PE placentas and the maternal circulation. ${ }^{63,64}$ This indicates a potential link between RARRES1 expression and apoptosis of VTs. Similarly to RARRES1, it was shown earlier that RXRA is significantly increased in placentas complicated by PE and IUGR. ${ }^{65}$ This suggests that RARRES1 expression might be dependent on RAR/RXR regulation. Interestingly, high syncytial RARRESI expression could also be detected in first-trimester placental tissue, implicating that increased RARRES1 in PE placentas might be due to a disturbed development of trophoblasts. Contrary to the finding that increased apoptosis rates are also reported in IUGR without PE, we did not detect an elevated RARRES1 expression in villous trophoblasts from IUGR placentas. This might be due to a less pronounced villous trophoblast apoptosis in IUGR. ${ }^{66}$ Furthermore, in contrast to IUGR trophoblasts, cultured trophoblasts from PE/IUGR placentas showed a significantly reduced syncytialisiation. ${ }^{67}$ So far, we cannot define whether the increased RARRES1 expression is a cause or a result of PE, but our results indicate it might be associated with villous trophoblast development. Furthermore, it has to be taken into account that our study focused on the analysis of idiopathic IUGR only. Thus, we currently cannot rule out a differential regulation of RARRESI secondary to various other etiopathologic causes of IUGR.

Both RARRES2 and 3 could only be detected in EVTs of the human third-trimester placenta. Interestingly, we observed the highest RARRES3 gene expression in primary trophoblast cells. This might partly be due to the high RARRES3 expression in placental blood cells, as the Percoll-gradient-based isolation of primary trophoblast cells contains about $2.4 \%$ to $4.5 \%$ 
mononuclear blood cells. ${ }^{14,68}$ RARRES3 is known to be a tumor suppressor in many cancers and thus influences proliferation, apoptosis, as well as cell adhesion. ${ }^{29,30,69,70}$ Consequently, we assume RARRES3 might be involved in the suppression of EVT proliferation and induction of apoptosis, thereby limiting their invasion. Additionally, RARRES3 is known to be involved in the regulation of epithelial-to-mesenchymal transition, where it, for example, induces adhesion via beta-catenin/cadherin, ${ }^{30}$ leading to EVT differentiation into placental bed giant cells. ${ }^{71}$ Such assumptions could support the detected pattern of RARRES3 expression.

RARRES2 is well described as a circulating maternal serum factor during pregnancy. ${ }^{25,72}$ Increased serum levels of RARRES2 were shown to be associated with the pregnancy complication PE. ${ }^{73,74}$ Our findings seem to be in contrast to these results, as we could not detect an increase of RARRES2 in

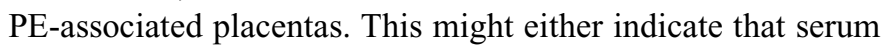
and tissue-specific RARRES2 concentrations differ, or, that the soluble form of RARRES2 originates from a source other than the placenta (e.g. adipocytes). For example, upregulation of RARRES2 expression was shown in postpregnancy mammary glands and thus might be associated with the indicated increased serum levels. ${ }^{75}$

Overall, we showed that trophoblast RARRES1, 2, and 3 expression could be induced via RARA, B, or G signaling. Even though RARB protein expression was not be detected within the nuclei of trophoblast cells, we showed that $R A R B$ expression in trophoblastic cell lines is dependent on RA induction and thus might also be involved in the regulation of gene expression. This is in line with former publications, which identified an RA responsive element within the $R A R B$ promotor. $^{34,53}$ In various carcinomas RA resistance was associated with loss of $R A R B$ expression. ${ }^{76}$ Here, we detected a loss of $R A R B$ expression in the choriocarcinoma cell lines, which could be significantly reversed by AZA treatment. Thus, we assume that $R A R B$ expression might also be dependent on promotor methylation in these cells. In contrast, we found high $R A R A$ and $R X R A$ expression in Jeg-3 cells, with $R X R A$ being the predominant receptor. This goes in line with an earlier publication showing high protein and RNA levels of both receptors in Jeg-3 cells. ${ }^{77}$ In placental tissues we detected a specific expression of RARA and RXRA in the villous and extravillous compartment. This was in line with former publications showing placental RARA and RXRA expressions. ${ }^{78-80}$ But in contrast to others, we were able to specify RXRA protein expression in the nuclei of VTs and not the SCT.

Taken together, placental RARRES1, 2, and 3 expression was detected in trophoblast cells of different placental compartments and could be induced in isolated trophoblast cells with RA derivatives via different RARs. These findings argue for a role of RARRES1, 2, and 3 for trophoblast function. Overexpression of RARRES1 in PE placentas might indicate that RARRES1 has some unfavorable effects contributing to the development of PE. As nutritional intake of vitamin A is a crucial factor during pregnancy, however, the PE-associated increase in placental RARRESI expression might suggest a possible therapeutic implication for dietary vitamin A restriction in women with severe PE.

\section{Acknowledgments}

We thank Jana Engelbrecht and Martin Fellermeier for their technical assistance and Dr G. Mor, Department of Obstetrics, Gynecology and Reproductive Sciences, Reproductive Immunology Unit, Yale University School of Medicine, USA, for the kind gift of Swan 71 cells. The present work was performed by Hanna Huebner in partial fulfillment of the requirements for obtaining the degree "Dr. rer. nat." at the Friedrich-Alexander-University Erlangen-Nuremberg (FAU), Germany.

\section{Author's Contribution}

The authors FB Fahlbusch and M. Ruebner contributed equally to this study.

\section{Declaration of Conflicting Interests}

The author(s) declared no potential conflicts of interest with respect to the research, authorship, and/or publication of this article.

\section{Funding}

The author(s) disclosed receipt of the following financial support for the research, authorship, and/or publication of this article: This work was supported by the ELAN (Erlanger Leistungsbezogene Anschubfinanzierung) program (13-06-14-1-Fahlbusch) to FF/MR and the Dr Robert Pfleger foundation (Bamberg, Germany) to FF/MR.

\section{Supplemental Material}

Supplemental material for this article is available online.

\section{References}

1. Chen M-C, Hsu S-L, Lin H, Yang T-Y. Retinoic acid and cancer treatment. Biomedicine (Taipei). 2014;4(4):22.

2. Nagpal S, Patel S, Asano AT, Johnson AT, Duvic M, Chandraratna RA. Tazarotene-induced gene 1 (TIG1), a novel retinoic acid receptor-responsive gene in skin. J Invest Dermatol. 1996;106(2): 269-274.

3. Cunningham TJ, Duester G. Mechanisms of retinoic acid signalling and its roles in organ and limb development. Nat Rev Mol Cell biol. 2015;16(2):110-123.

4. Rhinn M, Dollé P. Retinoic acid signalling during development. Development. 2012;139(5):843-858.

5. Das BC, Thapa P, Karki R, et al. Retinoic acid signaling pathways in development and diseases. Bioorg Med Chem. 2014;22(2): 673-683.

6. Maden M. Retinoid signalling in the development of the central nervous system. Nat Rev Neurosci. 2002;3(11):843-853.

7. Merlet-Bénichou C, Vilar J, Lelièvre-Pégorier M, Gilbert T. Role of retinoids in renal development: pathophysiological implication. Curr Opin Nephrol Hypertens. 1999;8(1):39-43.

8. Nagpal S, Chandraratna RA. Recent developments in receptorselective retinoids. Curr Pharm Des. 2000;6(9):919-931.

9. Allenby G, Bocquel M-T, Saunders M, et al. Retinoic acid receptors and retinoid $\mathrm{X}$ receptors: interactions with endogenous retinoic acids. Proc Natl Acad Sci U S A. 1993;90(1):30-34. 
10. Kato Y, Braunstein GD. Retinoic acid stimulates placental hormone secretion by choriocarcinoma cell lines in vitro. Endocrinology. 1991;128(1):401-407.

11. Stephanou A, Handwerger S. Retinoic acid and thyroid hormone regulate placental lactogen expression in human trophoblast cells. Endocrinology. 1995;136(3):933-938.

12. Tarrade A, Schoonjans K, Guibourdenche J, et al. PPAR $\gamma / R X R \alpha$ heterodimers are involved in human CG $\beta$ synthesis and human trophoblast differentiation. Endocrinology. 2001;142(10): 4504-4514.

13. Tarrade A, Schoonjans K, Pavan L, et al. PPAR $\gamma / R X R \alpha$ heterodimers control human trophoblast invasion. J Clin Endocrinol Metab. 2001;86(10):5017-5024.

14. Ruebner M, Langbein M, Strissel PL, et al. Regulation of the human endogenous retroviral Syncytin-1 and cell-cell fusion by the nuclear hormone receptors PPAR $\gamma / \mathrm{RXR} \alpha$ in placentogenesis. $J$ Cell Biochem. 2012;113(7):2383-2396.

15. Parast MM, Yu H, Ciric A, Salata MW, Davis V, Milstone DS. PPAR $\gamma$ regulates trophoblast proliferation and promotes labyrinthine trilineage differentiation. PLoS One. 2009;4(11):e8055.

16. Peng Z, Shen R, Li Y-W, Teng K-Y, Shapiro CL, Lin H-JL. Epigenetic repression of RARRES1 is mediated by methylation of a proximal promoter and a loss of CTCF binding. PloS One. 2012;7(5):e36891.

17. Jing C, El-Ghany MA, Beesley C, Foster CS, Rudland PS, Smith $\mathrm{P}$, et al. Tazarotene-induced gene 1 (TIG1) expression in prostate carcinomas and its relationship to tumorigenicity. J Natl Cancer Inst. 2002;94(7):482-490.

18. Oldridge EE, Walker HF, Stower MJ, et al. Retinoic acid represses invasion and stem cell phenotype by induction of the metastasis suppressors RARRES1 and LXN. Oncogenesis. 2013;2:e45.

19. Wu CC, Tsai FM, Shyu RY, Tsai YM, Wang CH, Jiang SY. G protein-coupled receptor kinase 5 mediates Tazarotene-induced gene 1-induced growth suppression of human colon cancer cells. BMC Cancer. 2011;11:175.

20. Ohnishi S, Okabe K, Obata H, et al. Involvement of tazaroteneinduced gene 1 in proliferation and differentiation of human adipose tissue-derived mesenchymal stem cells. Cell Prolif. 2009; 42(3):309-316.

21. Zabel BA, Allen SJ, Kulig P, et al. Chemerin activation by serine proteases of the coagulation, fibrinolytic, and inflammatory cascades. J Biol Chem. 2005;280(41):34661-34666.

22. Ernst MC, Sinal CJ. Chemerin: at the crossroads of inflammation and obesity. Trends Endocrinol Metab. 2010;21(11):660-667.

23. Ferland DJ, Watts SW. Chemerin: a comprehensive review elucidating the need for cardiovascular research. Pharmacol Res. 2015;99:351-361

24. Nagpal S, Patel S, Jacobe $\mathrm{H}$, et al. Tazarotene-induced gene 2 (TIG2), a novel retinoid-responsive gene in skin. J Invest Dermatol. 1997;109(1):91-95.

25. Garces MF, Sanchez E, Ruíz-Parra AI, et al. Serum chemerin levels during normal human pregnancy. Peptides. 2013;42: 138-143.

26. Erdogan S, Yilmaz FM, Yazici O, et al. Inflammation and chemerin in colorectal cancer. Tumor Biology. 2016;37(5): 6337-6342.
27. Szydło B, Kiczmer P, Świętochowska E, Ostrowska Z. Role of omentin and chemerin in metabolic syndrome and tumor diseases. Postepy Hig Med Dośw (Online). 2016;70(0):844-849.

28. Farsam V, Basu A, Gatzka M, et al. Senescent fibroblast-derived chemerin promotes squamous cell carcinoma migration. Oncotarget. 2016;7(50):83554-83569.

29. Morales M, Arenas EJ, Urosevic J, et al. RARRES3 suppresses breast cancer lung metastasis by regulating adhesion and differentiation. EMBO Mol Med. 2014;6(7):865-881.

30. Wang Z, Wang L, Hu J, et al. RARRES3 suppressed metastasis through suppression of MTDH to regulate epithelialmesenchymal transition in colorectal cancer. Am J Cancer Res. 2015;5(6):1988-1899.

31. Higuchi E, Chandraratna RA, Hong WK, Lotan R. Induction of TIG3, a putative class II tumor suppressor gene, by retinoic acid in head and neck and lung carcinoma cells and its association with suppression of the transformed phenotype. Oncogene. 2003; 22(30):4627-4635.

32. Shutoh M, Oue N, Aung PP, et al. DNA methylation of genes linked with retinoid signaling in gastric carcinoma: expression of the retinoid acid receptor beta, cellular retinol-binding protein 1, and tazarotene-induced gene 1 genes is associated with DNA methylation. Cancer. 2005;104(8):1609-1619.

33. Zhang J, Liu L, Pfeifer GP. Methylation of the retinoid response gene TIG1 in prostate cancer correlates with methylation of the retinoic acid receptor beta gene. Oncogene. 2004;23(12): 2241-2249.

34. de The H, del Mar Vivanco-Ruiz M, Tiollais P, Stunnenberg H, Dejean A. Identification of a retinoic acid responsive element in the retinoic acid receptor \& beta; gene. Nature. 1990;343(6254): 177-180.

35. Alholle A, Brini AT, Gharanei S, et al. Functional epigenetic approach identifies frequently methylated genes in ewing sarcoma. Epigenetics. 2013;8(11):1198-1204.

36. Mohammad N, Yaqinuddin A, Kakal F, Sheikh L, Qureshi R, Somani M. Frequent hypomethylation of PTGS2 gene promoter in human term placenta. Ital J Anat Embryol. 2013;118(2): 211-216.

37. Ferretti C, Bruni L, Dangles-Marie V, Pecking AP, Bellet D. Molecular circuits shared by placental and cancer cells, and their implications in the proliferative, invasive and migratory capacities of trophoblasts. Hum Reprod Update. 2007;13(2): 121-141.

38. Huppertz B. Placental origins of preeclampsia challenging the current hypothesis. Hypertension. 2008;51(4):970-975.

39. Bracken MB, Brinton LA, Hayashi K. Epidemiology of hydatidiform mole and choriocarcinoma. Epidemiol Rev. 1984;6: 52-75.

40. Fahlbusch FB, Dawood Y, Hartner A, et al. Cullin 7 and Fbxw 8 expression in trophoblastic cells is regulated via oxygen tension: implications for intrauterine growth restriction? J Matern Fetal Neonatal Med. 2012;25(11):2209-2215.

41. Ruebner M, Strissel PL, Ekici AB, et al. Reduced syncytin-1 expression levels in placental syndromes correlates with epigenetic hypermethylation of the ERVW-1 promoter region. PLoS One. 2013;8(2):e56145. 
42. Ruebner M, Strissel PL, Langbein M, et al. Impaired cell fusion and differentiation in placentae from patients with intrauterine growth restriction correlate with reduced levels of HERV envelope genes. J Mol Med (Berl). 2010;88(11):1143-1156.

43. Straszewski-Chavez SL, Abrahams VM, Alvero AB, et al. The isolation and characterization of a novel telomerase immortalized first trimester trophoblast cell line, Swan 71. Placenta. 2009; 30(11):939-948.

44. Yen A, Fenning R, Chandraratna R, Walker P, Varvayanis S. A retinoic acid receptor $\beta / \gamma$-selective prodrug (tazarotene) plus a retinoid $\mathrm{X}$ receptor ligand induces extracellular signal-regulated kinase activation, retinoblastoma hypophosphorylation, G0 arrest, and cell differentiation. Mol pharmacol. 2004;66(6): 1727-1737.

45. Chandraratna R. Tazarotene-first of a new generation of receptor-selective retinoids. Br J Dermatol. 1996;135(suppl 49):18-25.

46. Chandraratna RA. Tazarotene: the first receptor-selective topical retinoid for the treatment of psoriasis. J Am Acad Dermatol. 1997; 37(2 pt 3):S12-S17.

47. Bosch A, Bertran SP, Lu Y, et al. Reversal by RAR $\alpha$ agonist Am580 of c-Myc-induced imbalance in RAR $\alpha / \operatorname{RAR} \gamma$ expression during MMTV-Myc tumorigenesis. Breast Cancer Res. 2012; 14(4):R121.

48. Ancian P, Lenoir M, Michel S. Effects of CD 2665, a selective RAR $\beta, \gamma$ antagonist on the retinoid activity in human keratinocytes and fibroblasts in culture. J Inv Dermatol. 1997;5(108):817.

49. Somenzi G, Sala G, Rossetti S, Ren M, Ghidoni R, Sacchi N. Disruption of retinoic acid receptor alpha reveals the growth promoter face of retinoic acid. PloS one. 2007;2(9):e836.

50. Shrestha S, Kim S-Y, Yun Y-J, et al. Retinoic acid induces hypersegmentation and enhances cytotoxicity of neutrophils against cancer cells. Immunol Lett. 2017;182:24-29.

51. Lo H-M, Chen C-L, Yang C-M, et al. The carotenoid lutein enhances matrix metalloproteinase- 9 production and phagocytosis through intracellular ROS generation and ERK1/2, p38 MAPK, and RAR $\beta$ activation in murine macrophages. J Leukoc Biol. 2013;93(5):723-735.

52. Rouliera S, Rochette-Egly C, Rebut-Bonneton C, Porquet D, Evain-Brion D. Nuclear retinoic acid receptor characterization in cultured human trophoblast cells: effect of retinoic acid on epidermal growth factor receptor expression. Mol Cell Endocrinol. 1994;105(2):165-173.

53. Tang X-H, Gudas LJ. Retinoids, retinoic acid receptors, and cancer. Annu Rev Patho. 2011;6:345-364.

54. Jerónimo C, Henrique R, Hoque MO, et al. Quantitative RARß2 Hypermethylation A Promising Prostate Cancer Marker. Clin Cancer Res. 2004;10(12 pt 1):4010-4014.

55. Bastian PJ, Ellinger J, Heukamp LC, Kahl P, Müller SC, von Rücker A. Prognostic value of $\mathrm{CpG}$ island hypermethylation at PTGS2, RAR-beta, EDNRB, and other gene loci in patients undergoing radical prostatectomy. Eur Urol. 2007;51(3): 665-674.

56. Jha A, Nikbakht M, Parashar G, Shrivastava A, Capalash N, Kaur J. Reversal of Hypermethylation and Reactivation of the RAR [Beta] 2 Gene by Natural Compounds in Cervical Cancer Cell Lines. Folia Biol(Praha). 2010;56(5):195-200.
57. Wang X, Saso H, Iwamoto T, et al. TIG1 Promotes the Development and Progression of Inflammatory Breast Cancer through Activation of Axl Kinase. Cancer Res. 2013;73(21): 6516-6525.

58. Wu CC, Shyu RY, Chou JM, et al. RARRES1 expression is significantly related to tumour differentiation and staging in colorectal adenocarcinoma. Eur J Cancer. 2006;42(4):557-565.

59. Yanatatsaneejit $\mathrm{P}$, Chalermchai $\mathrm{T}$, Kerekhanjanarong V, et al. Promoter hypermethylation of CCNA1, RARRES1, and HRASLS3 in nasopharyngeal carcinoma. Oral oncology. 2008; 44(4):400-406.

60. Coyle K, Murphy J, Vidovic D, et al. Breast cancer subtype dictates DNA methylation and ALDH1A3-mediated expression of tumor suppressor RARRES1. Oncotarget. 2016;7(28): 44098-44112.

61. Langbein M, Strick R, Strissel PL, et al. Impaired cytotrophoblast cell-cell fusion is associated with reduced Syncytin and increased apoptosis in patients with placental dysfunction. Mol Reprod Dev. 2008;75(1):175-183.

62. Gauster M, Moser G, Orendi K, Huppertz B. Factors involved in regulating trophoblast fusion: potential role in the development of preeclampsia. Placenta. 2009;30(suppl A):S49-S54.

63. Heazell A, Moll S, Jones C, Baker P, Crocker I. Formation of syncytial knots is increased by hyperoxia, hypoxia and reactive oxygen species. Placenta. 2007;28(suppl A):S33-S40.

64. Burton GJ, Jones CJ. Syncytial knots, sprouts, apoptosis, and trophoblast deportation from the human placenta. Taiwan $J$ Obstet Gynecol. 2009;48(1):28-37.

65. Holdsworth-Carson S, Lim R, Mitton A, et al. Peroxisome proliferator-activated receptors are altered in pathologies of the human placenta: gestational diabetes mellitus, intrauterine growth restriction and preeclampsia. Placenta. 2010;31(3): 222-219.

66. Longtine MS, Chen B, Odibo AO, Zhong Y, Nelson DM. Villous trophoblast apoptosis is elevated and restricted to cytotrophoblasts in pregnancies complicated by preeclampsia, IUGR, or preeclampsia with IUGR. Placenta. 2012;33(5):352-359.

67. Newhouse SM, Davidge ST, Winkler-Lowen B, Demianczuk N, Guilbert LJ. In Vitro Differentiation of Villous Trophoblasts from Pregnancies Complicated by Intrauterine Growth Restriction With and Without Pre-Eclampsia. Placenta. 2007;28(10): 999-1003.

68. Fahlbusch FB, Ruebner M, Volkert G, et al. Corticotropinreleasing hormone stimulates expression of leptin, 11 betaHSD2 and syncytin-1 in primary human trophoblasts. Reprod Biol Endocrinol. 2012;10(1):80.

69. Shyu R, Jiang S, Chou J, et al. RARRES3 expression positively correlated to tumour differentiation in tissues of colorectal adenocarcinoma. Br J Cancer. 2003;89(1):146-151.

70. Casanova B, de la Fuente MT, Garcia-Gila M, et al. The class II tumor-suppressor gene RARRES3 is expressed in B cell lymphocytic leukemias and down-regulated with disease progression. Leukemia. 2001;15(10):1521-1526.

71. Li HW, Cheung AN, Tsao SW, Cheung AL, WS O. Expression of e-cadherin and beta-catenin in trophoblastic tissue in normal and pathological pregnancies. Int J Gynecol Pathol. 2003;22(1):63-70. 
72. Kasher-Meron M, Mazaki-Tovi S, Barhod E, et al. Chemerin concentrations in maternal and fetal compartments: implications for metabolic adaptations to normal human pregnancy. $J$ Perinat Med. 2014;42(3):371-378.

73. Stepan H, Philipp A, Roth I, et al. Serum levels of the adipokine chemerin are increased in preeclampsia during and 6months after pregnancy. Regul Pept. 2011;168(1):69-72.

74. Duan DM, Niu JM, Lei Q, Lin XH, Chen X. Serum levels of the adipokine chemerin in preeclampsia. J Perinat Med. 2012;40(2): 121-127.

75. Verlinden I, Güngör N, Janssens J, Michiels L. Gene expression profiling to identify parity-induced changes in the human mammary gland. Breast Cancer Res. 2005;7:1.

76. Xu X-C. Tumor-suppressive activity of retinoic acid receptor- $\beta$ in cancer. Cancer Lett. 2007;253(1):14-24.
77. Guibourdenche J, Roulier S, Rochette-Egly C, Evain-Brion D. High retinoid $\mathrm{X}$ receptor expression in JEG-3 choriocarcinoma cells: involvement in cell function modulation by retinoids. $J$ Cell Physiol. 1998;176(3):595-601.

78. Guibourdenche J, Alsat E, Soncin F, Rochette-Egly C, EvainBrion D. Retinoid receptors expression in human term placenta: involvement of RXR $\alpha$ in retinoid induced-hCG secretion. J Clin Endocrinol Metab. 1998;83(4):1384-1387.

79. Rodie VA, Young A, Jordan F, Sattar N, Greer IA, Freeman D. Human placental peroxisome proliferator-activated receptor $\delta$ and $\gamma$ expression in healthy pregnancy and in preeclampsia and intrauterine growth restriction. J Soc Gynecol Investig. 2005;12(5):320-329.

80. Tarrade A, Rochette-Egly C, Guibourdenche J, Evain-Brion D. The expression of nuclear retinoid receptors in human implantation. Placenta. 2000;21(7):703-710. 